\title{
Spin-Dependent Coupling of Charged Quantum Dot Excitons with Continuum States
}

\author{
B. Urbaszek ${ }^{a, b}$, R.J. WARBUrton ${ }^{a}$, K. KARrai ${ }^{c}$, \\ C. Schulhauser ${ }^{c}$, A. HöGele ${ }^{c}$, E.J. McGheE $^{a}$, A.O. Govorov ${ }^{d}$, \\ B.D. Gerardot ${ }^{e}$, X. MArie ${ }^{b}$, T. Amand $^{b}$ And P.M. PetrofF ${ }^{e}$ \\ ${ }^{a}$ School of Engineering and Physical Sciences, Heriot-Watt University \\ Edinburgh EH14 4AS, UK \\ ${ }^{b}$ Laboratoire de Nanophysique, Magnétisme et Optoélectronique, INSA \\ 135 av. de Rangueil, 31077 Toulouse Cedex 4, France \\ ${ }^{c}$ Center for NanoScience and Sektion Physik, Ludwig-Maximilians-Universität \\ Geschwister-Scholl-Platz 1, 80539 München, Germany \\ ${ }^{d}$ Department of Physics and Astronomy, Ohio University, Athens, Ohio USA \\ ${ }^{e}$ Materials Department, University of California \\ Santa Barbara, California 93106, USA
}

\begin{abstract}
Magnetic field and temperature dependent photoluminescence studies on neutral and charged excitons in individual InAs quantum dots allow us to uncover different mechanisms by which the discrete quantum dot states are coupled to delocalized continuum states in a quantum well (the wetting layer). The behaviour of the neutral and singly charged excitons can be explained taking only discrete quantum dot states into account. For doubly and triply charged excitons we have to consider spin dependent coherent and incoherent interactions between discrete quantum dot states and delocalized wetting layer states.
\end{abstract}

PACS numbers: 73.21.La, 78.67.Hc

\section{Introduction}

The discrete nature of optical transitions in individual semiconductor quantum dots can be described by an artificial atom model and has stimulated proposals for using dots in quantum information devices. This model can be justified by the 
shell structure observed when a quantum dot is filled either with excitons [1] or with electrons [2]. At low temperature, the artificial atom picture is strengthened by the long coherence times of an exciton in a quantum dot [3-5], motivating the application of quantum dots in quantum optics and quantum information processing. Excitons in quantum dots have already been manipulated coherently [6-8]. Whereas interactions of discrete quantum dot states and continuum states from the wetting layer are usually regarded as a source of incoherence [9], they can also give rise to a new type of coherent interaction [10].

Here we report magnetic field ( 0 to $9 \mathrm{~T}$ ) and temperature dependent (4 to $30 \mathrm{~K}$ ) photoluminescence (PL) on neutral and charged excitons in individual InAs quantum dots. Our approach is to compare configurations with different occupations of $s$ - and $p$-like electron levels. We do this by adding excess electrons to the neutral exciton $\mathrm{X}^{0}$ to create the singly charged exciton $\mathrm{X}^{1-}$ (trion), the doubly charged exciton $\mathrm{X}^{2-}$ and the triply charged exciton $\mathrm{X}^{3-}$. We employ quantum dots embedded in a field-effect structure that allows us to control precisely the charge of the exciton [2]. In these experiments, a hole is generated with optical excitation. Over large regions of bias voltage, the excitonic charge is constant, and there are abrupt steps in emission energy whenever an additional electron is added to the dot [2]. The sample is grown on a GaAs substrate, on which a buffer layer, an $n^{+}$-GaAs layer (the back contact) and then a $25 \mathrm{~nm}$ thick undoped GaAs

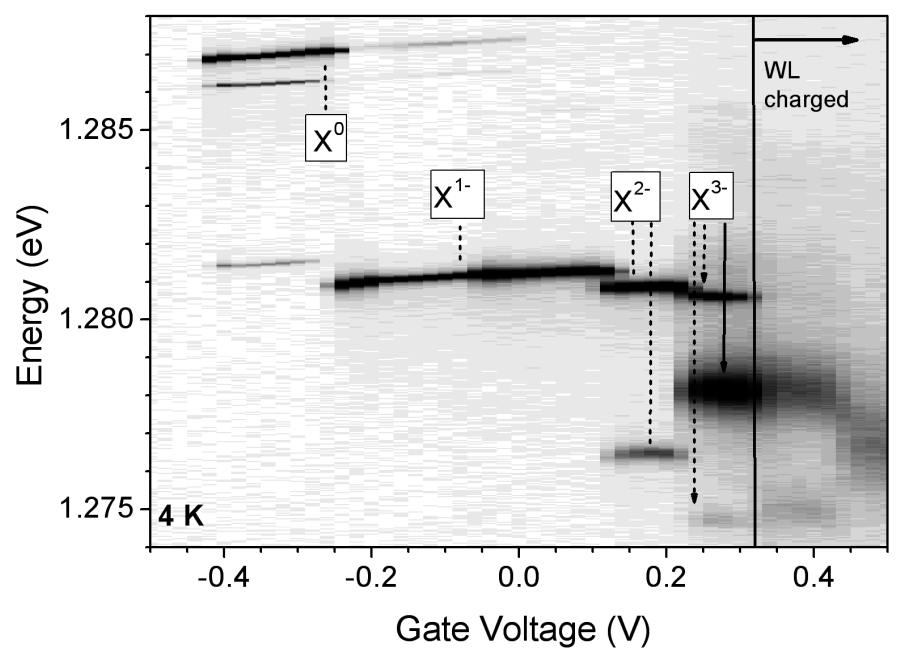

Fig. 1. Grey-scale plot of the photoluminescence versus gate voltage for a single dot from sample $A$ at $4.2 \mathrm{~K}$. White corresponds to 0 counts, black to 1000 counts on the detector. The excitons are labelled with $\mathrm{X}$ standing for exciton, with excess charge as the suffix. Above a voltage of about $0.32 \mathrm{~V}$, the wetting layer (WL) loads with electrons. The $\mathrm{X}^{3-}$ transitions with an initial spin $S=1(S=0)$ are marked by a dotted (solid) arrow. The unlabelled weaker PL lines are related to biexciton emission. 
layer (the tunnel barrier) are grown. Subsequently, the InAs quantum dots are formed in the Stranski-Krastanow growth mode, with a density in the range of $10^{9} \mathrm{~cm}^{-2}$. A GaAs/AlAs superlattice (the blocking barrier), grown on top of the GaAs covering the dots, ensures that the coupling between the dots and a surface gate electrode is purely capacitive. The dots have an ensemble PL centred at either $1.3 \mathrm{eV}$ (sample $A$ ) or at $1.1 \mathrm{eV}$ (sample $B$ ) depending on the growth. We have performed PL spectroscopy of individual quantum dots as a function of gate voltage $V_{\mathrm{g}}$ at different temperatures using a confocal microscope. The PL is excited with an $850 \mathrm{~nm}$ laser diode, and is dispersed and detected with a spectrometer-Si-CCD system. The magnetic field is applied perpendicular to the quantum dot plane.

At low laser power, for each individual dot, we can identify $\mathrm{X}^{0}, \mathrm{X}^{1-}, \mathrm{X}^{2-}$, and $\mathrm{X}^{3-}$ by the large jump in $\mathrm{PL}$ energy on going from $\mathrm{X}^{0}$ to $\mathrm{X}^{1-}$, and the characteristic splittings of $\mathrm{X}^{2}$ and $\mathrm{X}^{3-}$, as shown in Fig. 1 for a dot from sample $A$.

\section{The neutral exciton $\mathrm{X}^{0}$ and the trion $\mathrm{X}^{1-}$}

In a magnetic field the behaviour of the $\mathrm{X}^{0}$ and $\mathrm{X}^{1-}$ is typical of localized excitons [11]. There is a diamagnetic shift, quadratic in magnetic field $B$, arising from the enhancement of the exciton confinement, and a splitting, linear in $B$, arising from the spin Zeeman effect, see Fig. 2a. Also the temperature dependence of the single dot line width is very similar for the two transitions, see Fig. 3a to d. Taking into account the spectral resolution of our experiment we obtain for the neutral exciton $\mathrm{X}^{0}$ a line width $\Gamma$ of $10 \pm 20 \mu \mathrm{eV}$ at $4.2 \mathrm{~K}$, in good agreement with a value of $2 \mu \mathrm{eV}$ obtained in a transmission experiment with a narrow

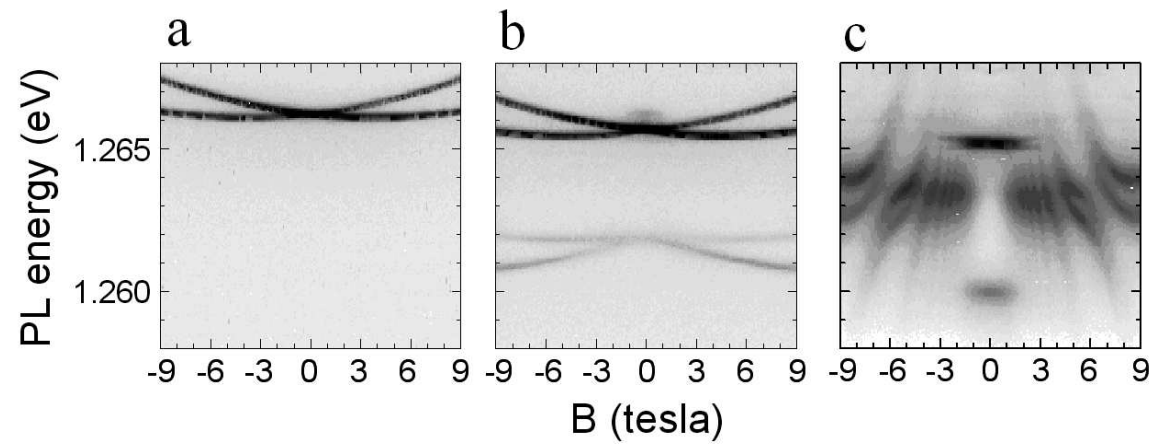

Fig. 2. PL from charged excitons from a different dot to Fig. 1. Data are shown for singly (a), doubly (b) and triply (c) charged excitons, all from the same quantum dot, versus magnetic field. The PL is represented with a grey scale, and the magnetic field is applied perpendicular to the quantum dot plane. The neutral exciton $\mathrm{X}^{0}$ is not shown because the shift and splitting in magnetic field are identical to those of the singly charged exciton. The $\mathrm{X}^{2-} \mathrm{PL}$ has two lines as a consequence of exchange-split final states, one with electron spin $S=1$, the other with $S=0$. 

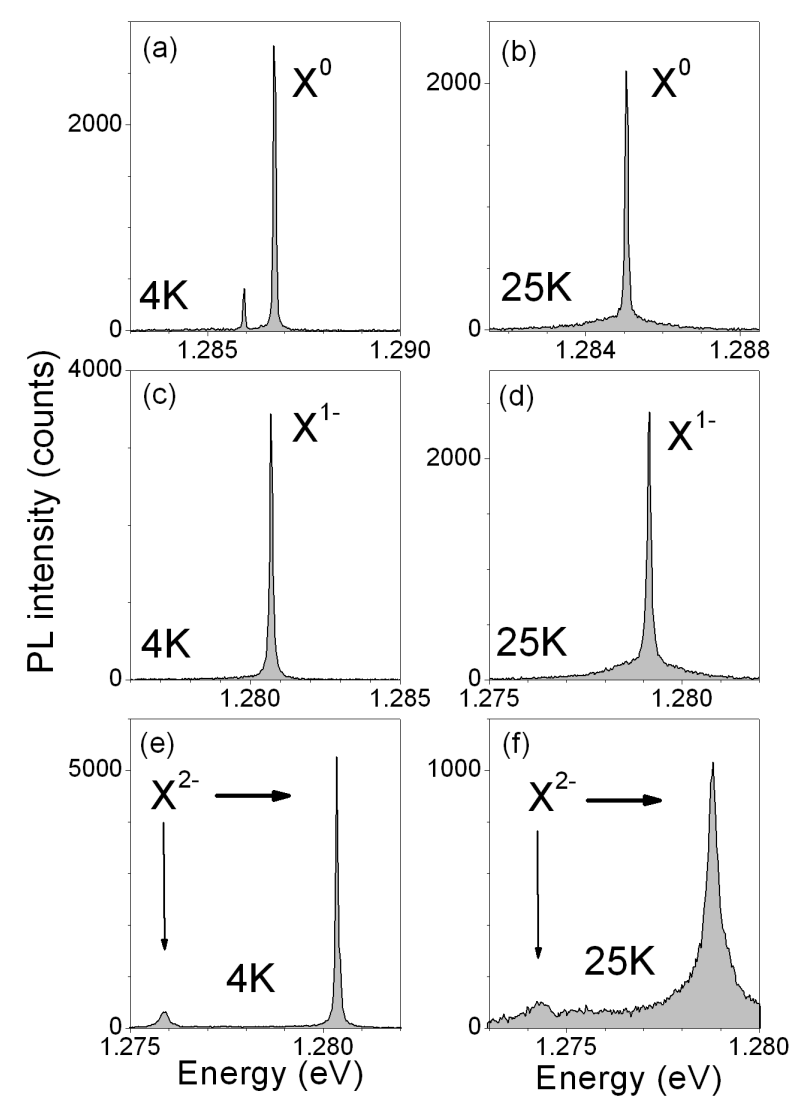

Fig. 3. $\mathrm{PL}$ of individual exciton transitions from sample $A$. (a) The neutral exciton $\mathrm{X}^{0}$ at $4 \mathrm{~K}$, the smaller peak on the low energy side is attributed to the biexciton; (b) $\mathrm{X}^{0}$ at $25 \mathrm{~K},(\mathrm{c})$ the $\mathrm{X}^{1-}$ transition at $4 \mathrm{~K}$, and (d) $\mathrm{X}^{1-}$ at $25 \mathrm{~K}$; (e) the $\mathrm{X}^{2-}$ transition at $4 \mathrm{~K}$, and (f) at $25 \mathrm{~K}$. Arrows indicate the emission into the $S=1(S=0)$ final state at high (low) energy.

line width laser [12]. This value increases only by a few $\mu \mathrm{eV}$ as the temperature is raised to $30 \mathrm{~K}$. The main effect of the temperature increase on the emission lines is the strong deviation from a Lorentzian line shape at higher temperature, see Fig. $3 \mathrm{~b}$ and $\mathrm{d}$, that we attribute to lattice relaxation due to exciton-acoustic phonon coupling [13].

\section{The doubly charged exciton $\mathrm{X}^{2-}$}

For $\mathrm{X}^{2-}$ at zero magnetic field there are two final states after recombination: a triplet with a total electron spin of $S=1$ at higher energy $(\mathrm{FWHM}<0.05 \mathrm{meV}$ at $4.2 \mathrm{~K}$ ) and a singlet $S=0$ at lower energy (FWHM $\approx 0.5 \mathrm{meV}$ at $4.2 \mathrm{~K}$ ), as can be seen in Fig. 3e. The two transitions are split by the electron-electron exchange 
energy $2 \mathrm{X}_{s p}^{\mathrm{ee}}[14]$ where $\mathrm{X}_{s p}^{\mathrm{ee}}$ is the exchange interaction energy between an electron in the $s$ state and an electron in the $p$ state. At low temperature, recombination into the singlet state has a noticeably larger line width than recombination into the triplet state, that we interpret as a spin dependence of the relaxation mechanism for the $p$ electron in the $\mathrm{X}^{2-}$ final state. The singlet-triplet splitting is typically $4 \mathrm{meV}[2,15,16]$ (sample $A$ ) and $7 \mathrm{meV}$ (sample $B$ ). Recent experiments also show an additional fine structure: the triplet is not a single line, but a doublet separated by the electron-hole exchange energy of $\approx 0.2 \mathrm{meV}$ in sample $B$ and $\approx 0.1 \mathrm{meV}$ in sample $A[17]$.

In a magnetic field we observe for the singlet and triplet transitions a diamagnetic shift and a Zeeman splitting typical of localized excitons, see Fig. 2b, as for $\mathrm{X}^{0}$ and $\mathrm{X}^{1-}[11]$. The line width for both triplet and singlet $\mathrm{X}^{2-}$ emission increases surprisingly rapidly with temperature at a rate much larger than for $\mathrm{X}^{0}$ and $\mathrm{X}^{1-}$. We suggest that this broadening is due to scattering of the carriers with acoustic phonons in the final state after photon emission, where this scattering is enhanced due to a low energy tail of the continuum that overlaps with the $p$ states [18].

\section{The triply charged exciton $\mathrm{X}^{3-}$}

For the dots of samples $A$ and $B$ the $p$ states are the highest confined electron states for our dots, as the $\mathrm{X}^{3-}$ is the most highly charged exciton that we can create, the $d$ states are not confined. For $\mathrm{X}^{3-}$, we observe, as for $\mathrm{X}^{2-}$, a splitting of the PL due to electron-electron exchange of the order of $7 \mathrm{meV}$, see transitions marked with dashed arrows in Fig. 1. This proves that the two $p$ electrons in the $\mathrm{X}^{3-}$ ground state have parallel spins $(S=1)$, with one electron in the $p_{x}$ state, the other in the $p_{y}$ state. The final states have $S=3 / 2$ or $S=1 / 2$ split by $3 \mathrm{X}_{s p}^{\mathrm{ee}}[2]$. For the optical transition from the $S=1$ to the $S=3 / 2$ state we can resolve a fine structure with three equidistant lines originating from electron-hole exchange effects in the initial state [17]. This fine structure only occurs for the $S=1$ initial state, there is no electron-hole exchange for the $S=0$ initial state. If the energy separation between $p_{x}$ and $p_{y}$ is larger than the exchange energy $\mathrm{X}_{p x p y}^{\mathrm{ee}}$, the $p_{x}$ orbital is doubly occupied such that $S=0$. In the case of an $S=0$ $\mathrm{X}^{3-}$, the PL consists of a single line between the singlet and triplet states of the $\mathrm{X}^{2-}$ emission [15]. In Fig. 1 we can see contributions from both $S=0$ and $S=1$ initial case, demonstrating that these two configurations are close in energy. The dot presented in Fig. 1 is presumably slightly more asymmetric in the quantum dot plane than the dot for which we present the magnetic field results in Fig. 2.

In a magnetic field, the $\mathrm{X}^{3-}$ has a behaviour that is completely different from the other transitions. At high field, in addition to the spin Zeeman effect, the PL develops a remarkable series of oscillations. This is preceded by a gradual collapse around $1 \mathrm{~T}$ of the two low field lines into a single line. A magnetic 
field introduces an orbital Zeeman splitting between the two $p$ orbitals, such that when the splitting between the $p$ orbitals exceeds the exchange energy, it becomes energetically favourable for both $p$ electrons to occupy the lower energy $p$ orbital and form an $S=0$ state. We attribute the collapse of the PL splitting around $1 \mathrm{~T}$ to this change in configuration [10].

In a magnetic field above about $1 \mathrm{~T}$, the $S=0$ initial state of the $\mathrm{X}^{3-}$ is favoured (two antiparallel spins in the $p_{x}$ state). In this case, the emission has a very large line width. We argue that the final state of the $S=0 \mathrm{X}^{3-}$ is responsible for the broadening as it couples efficiently to the energetically higher lying continuum states. For weakly confined $p$ states, this coupling initially introduces incoherence. At high magnetic fields Landau levels form in the continuum of wetting layer states. The discrete quantum dot states can couple coherently to these Landau levels giving rise to a series of anticrossings. The coupling occurs via Auger-like processes and the optical spectra can be described with an Anderson-like Hamiltonian by including the interaction between localized exciton states and delocalized states of the wetting layer [19].

Occupation of the wetting layer is found to promote the $S=0 \mathrm{X}^{3-}$ over the $S=1$, analogous to a magnetic field. In this case, the $\mathrm{X}^{3-}$ emission becomes broad with dot- and energy-dependent features. The temperature dependence of the PL

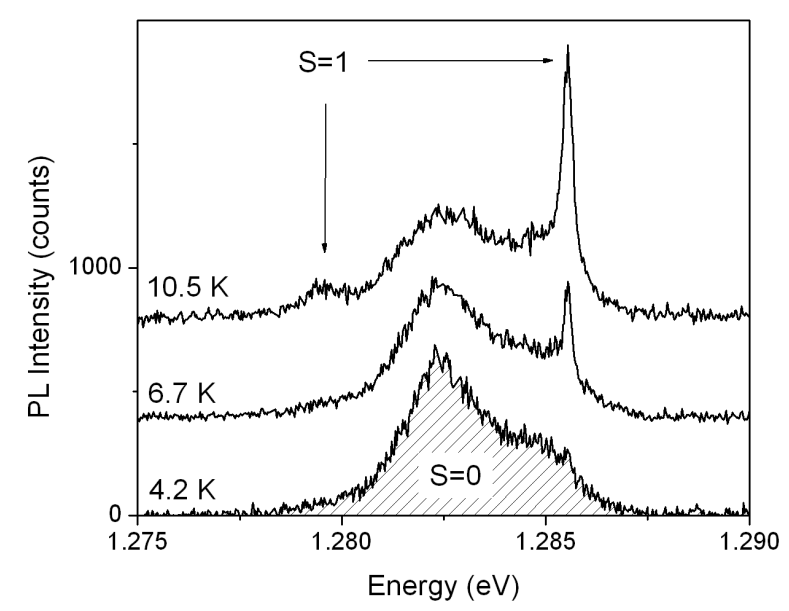

Fig. 4. Temperature dependence of the $\mathrm{X}^{3-}$ emission with an occupied wetting layer. No magnetic field is applied and the curves for the three different temperatures are offset for clarity. This experiment was performed on a different dot to Figs. 1-3. Occupation of the wetting layer favours the $S=0$ initial state and its emission gives the broad band. On increasing the temperature, the $S=1$ initial state is thermally occupied, leading to the emission at $1.286 \mathrm{eV}(1.2795 \mathrm{eV})$ into the $S=3 / 2(S=1 / 2)$ final states, as indicated by arrows. Let us note that the emission into the $S=1 / 2$ final state is broad even without an occupied wetting layer [2] because the final state can relax without a spin-flip. 
from an $\mathrm{X}^{3-}$ with occupied wetting layer is shown in Fig. 4. At low temperature, there is a very broad line, but, remarkably, on increasing the temperature, a sharp line emerges. The broad PL peak arises from the $S=0 \mathrm{X}^{3-}$ broad band because the hybridization with the continuum allows the final state in the absence of a magnetic field to decay rapidly. We identify the sharp line as emission from the thermally occupied $S=1$ initial state into the $S=3 / 2$ final state both from its energy and from the emergence of its partner $(S=1 / 2$ final state) at lower energy. The $S=0$ and $S=1$ excitons have almost degenerate initial states and there is no obvious reason why the initial states should have different energy broadenings. But there is a crucial difference in the final states: the $S=0 \mathrm{X}^{3-}$ final state hybridizes with the continuum, the $S=1 \mathrm{X}^{3-}$ final states do not. The absence of hybridization allows the $S=1 \mathrm{X}^{3-}$ exciton to retain a sharp PL despite the massive broadening of the $S=0 \mathrm{X}^{3-} \mathrm{PL}$.

\section{Conclusions}

We have employed charge tunable structures to study the coupling of discrete quantum dot levels with continuum states in the wetting layer. The behaviour of the exciton $\mathrm{X}^{3-}$ differs strongly from the other exciton states as it forms a coherent hybridization with Landau levels in the wetting layer.

\section{Acknowledgments}

We acknowledge discussions with A. Rosch, J. von Delft, and K.V. Kavokin. This work was funded by the DFG, EPSRC, The Royal Society and AC "Nanosciences-Nanotechnologies".

\section{References}

[1] M. Bayer, O. Stern, P. Hawrylak, S. Fafard, A. Forchel, Nature (Lond.) 405, 923 (2000).

[2] R.J. Warburton, C. Schäflein, D. Haft, F. Bickel, A. Lorke, K. Karrai, J. Garcia, W. Schoenfeld, P.M. Petroff, Nature (Lond.) 405, 926 (2000).

[3] D. Gammon, E.S. Snow, B.V. Shanabrook, D.S. Katzer, D. Park, Science 273, 87 (1996).

[4] P. Borri, W. Langbein, S. Schneider, U. Woggon, R.L. Sellin, D. Ouyang, D. Bimberg, Phys. Rev. Lett. 87, 157401 (2001).

[5] M. Bayer, A. Forchel, Phys. Rev. B 65, 041308 (2002).

[6] N.H. Bonadeo, J. Erland, D. Gammon, D. Park, D.S. Katzer, D.G. Steel, Science 282, 1473 (1998).

[7] H. Kamada, H. Gotoh, J. Temmyo, T. Takagahara, H. Ando, Phys. Rev. Lett. 87, 246401 (2001). 
[8] A. Zrenner, E. Beham, S. Stufler, F. Findels, M. Bichler, G. Abstreiter, Nature 418, 612 (2002).

[9] C. Kammerer, G. Cassabois, C. Voisin, C. Delalande, Ph. Roussignol, J.M. Gerard, Phys. Rev. Lett. 87, 207401 (2001).

[10] K. Karrai, R.J. Warburton, C. Schulhauser, A. Högele, B. Urbaszek, E.J. McGhee, A.O. Govorov, J.M. Garcia, B.D. Gerardot, P.M. Petroff, Nature 427, 135 (2004).

[11] C. Schulhauser, D. Haft, R.J. Warburton, K. Karrai, A.O. Govorov, A.V. Kalameitsev, A. Chaplik, W. Schoenfeld, J. Garcia, P.M. Petroff, Phys. Rev. B 66, 193303 (2002).

[12] A. Hogele, B. Alen, F. Bickel, R.J. Warburton, P.M. Petroff, K. Karrai, Physica E 21, 175 (2004).

[13] L. Besombes, K. Kheng, L. Marsal, H. Mariette, Phys. Rev. B 63, 155307 (2001).

[14] A. Wojs, P. Hawrylak, Phys. Rev. B 55, 13066 (1997).

[15] J.J. Finley, P.W. Fry, A.D. Ashmore, A. Lemaitre, A.I. Tartakovskii, R. Oulton, D.J. Mowbray, M.S. Skolnick, M. Hopkinson, P.D. Buckle, P.A. Maksym, Phys. Rev. B 63, 161305 (2001)

[16] F. Findeis, M. Baier, A. Zrenner, M. Bichler, G. Abstreiter, U. Hohenester, E. Molinari, Phys. Rev. B 63, 121309 (2001).

[17] B. Urbaszek, R.J. Warburton, K. Karrai, B.D. Gerardot, P.M. Petroff, J.M. Garcia, Phys. Rev. Lett. 90, 247403 (2003).

[18] B. Urbaszek, E.J. McGhee, M. Krüger, R.J. Warburton, K. Karrai, T. Amand, B.D. Gerardot, P.M. Petroff, J.M. Garcia, Phys. Rev. B 69, 035304 (2004).

[19] A.O. Govorov, K. Karrai, R.J. Warburton, A.V. Kalameitsev, Physica E 20, 295 (2004). 